\title{
Propagation of spinning acoustic modes in partially choked converging ducts
}

\author{
A. H. Nayfeh, J. J. Kelly, and L. T. Watson \\ Virginia Polytechnic Institute and State University, Blacksburg, Virginia 24061 \\ (Received 6 February 1981; accepted for publication 1 November 1981)
}

\begin{abstract}
A computer model based on the wave-envelope technique is used to study the propagation of spinning acoustic modes in converging hard-walled and lined circular ducts carrying near sonic mean flows. The results show that with increasing spinning mode number the intensification of the acoustic signal at the throat decreases for upstream propagation. The influence of the throat Mach number, frequency, boundary-layer thickness, and liner admittance on the propagation of spinning modes is considered.
\end{abstract}

PACS numbers: 43.20.Mv, 43.20.Bi, 43.50.Nm, 43.85.Ta

\section{INTRODUCTION}

The use of high bypass turbofan engines has resulted in the reduction of noise associated with the jet exhaust and has enhanced the fuel economy of the engine. But noise emissions from the inlet nacelles produced by the fan are of such a level as to raise objections from communities near airports. Hence much attention has been devoted to reducing the inlet noise. The use of choked inlets has long been recognized as an effective means of reducing upstream noise propagation ${ }^{1,2}$ although such inlets require careful design to prevent excessive loss in compressor performance. Therefore using a near sonic inlet, or partially choked inlet, along with an acoustic duct liner is one method that has received considerable attention in the reduction of inlet noise. In a previous analysis, $\mathrm{Nayfeh}^{3}$ used the wave-envelope method to study the propagation of axisymmetric modes. The wave-envelope method $^{4,5}$ is used in this report to study the propagation of spinning acoustic modes in the near sonic region of a simple converging duct section. The results show the effect of spinning mode number, Mach number, duct-liner admittance, frequency, and boundary-layer thickness. Both upstream and downstream propagation are considered. From this analysis it appears that the spinning modes also are refracted and intensified for upstream propagation in the throat region but not to the same degree as axisymmetric modes. The intensification of the acoustic pressure amplitude increases with increasing throat Mach number, frequency, and boundary layer thickness.

Several analytical as well as numerical techniques have been developed for the analysis of wave propagation in uniform and nonuniform ducts. Surveys of these techniques were made by Nayfeh et al., ${ }^{6}$ Nayfeh, ${ }^{7}$ and Nayfeh et $a l^{4}$ In addition to the wave-envelope technique, ${ }^{4,5}$ three finite-element methods ${ }^{8-10}$ were developed for the study of transmission of sound in variable-area ducts with compressible flow. The first method ${ }^{8}$ employs a velocity-potential formulation of the basic equations and thus it is restricted to irrotational mean flows. The others are more general in the sense that they permit the consideration of rotational mean flows. Eversman ${ }^{9}$ compared transmission and reflection coefficients obtained from a finite-element procedure with those from the method of weighted residuals and found good agreement. Although the mean-flow model is ro- tational, it apparently does not include the refractive effect of a finite boundary-layer thickness at the duct walls. The finite-element method of Abrahamson ${ }^{10}$ was developed for a general compressible mean flow but it is currently implemented only for an incompressible model. Comparisons between results of this finiteelement procedure and the wave-envelope method were carried out for cases of low-speed mean flows. ${ }^{4.5}$ These comparisons are of acoustic pressure distributions since the finite-element program was not written to obtain transmission and reflection coefficients. The agreement is very good.

A résumé of the wave-envelope technique is contained in the next section, whereas the results are presented in Sec. II.

\section{THE WAVE-ENVELOPE TECHNIQUE}

The wave-envelope technique is used to study acoustic propagation in hard- and soft-walled circular ducts (Fig. 1) carrying viscous or inviscid compressible mean flows. The mean flow is subsonic and the axial and radial gradients of the mean flow are not small. The cross section of the duct varies with the axial distance.

The reference values used to nondimensionalize the governing equations are the speed of sound $c_{a}$ in the uniform duct, the radius $R_{0}$ of the uniform duct, and $R_{0} / c_{a}$ (the reference time). The pressure $p$ is nondimensionalized by using $\rho_{a} c_{a}^{2}$, the density $\rho$ and temperature $T$ are nondimensionalized by using their corresponding reference values, and the viscosity $\mu$ and the thermal conductivity $\kappa$ are nondimensionalized by using their wall values in the uniform section.

A steady, sheared mean flow is assumed in the model and the presence of sound in the duct results in the

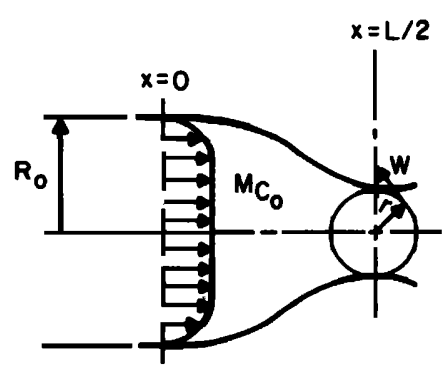

FIG. 1. Flow configuration. 
perturbation of the flow quantities so that

$$
q(\mathbf{r}, t)=q_{0}(\mathbf{r})+q_{1}(\mathbf{r}, t) .
$$

In this equation $q$ stands for any flow quantity, $r$ is the position vector, $t$ is time, $q_{0}$ is the mean part, and $q_{1}$ is the acoustic disturbance. In terms of the cylindrical coordinate system $(r, \theta, x)$, each acoustic quantity $q_{1}(r, \theta, x)$ is expressed for sinusoidal time variations as

$$
q_{1}(r, x, \theta, t)=\sum_{m=0}^{\infty} q_{1 m}(r, x) \exp [-i(\omega t-m \theta)],
$$

where $\omega$ is the dimensionless frequency. Then, the acoustic equations in cylindrical coordinates are (assuming the acoustic disturbance is inviscid and the nonlinear terms are negligible)

$$
\begin{aligned}
& -i \omega \rho_{1}+\frac{\partial}{\partial x}\left(\rho_{0} u_{1}+u_{0} \rho_{1}\right)+\frac{i \rho_{0} m}{r} w_{1}+\frac{1}{r} \frac{\partial}{\partial r} \\
& \quad \times\left(r \rho_{0} v_{1}+r v_{0} \rho_{1}\right)=0 \\
& \rho_{0}\left(-i \omega u_{1}+\frac{\partial}{\partial x}\left(u_{0} u_{1}\right)+v_{0} \frac{\partial u_{1}}{\partial r}+v_{1} \frac{\partial u_{0}}{\partial r}\right)+\rho_{1}\left(u_{0} \frac{\partial u_{0}}{\partial x}\right. \\
& \left.\quad+v_{0} \frac{\partial u_{0}}{\partial r}\right)=-\frac{\partial p_{1}}{\partial x}, \\
& \rho_{0}\left(-i \omega v_{1}+\frac{\partial}{\partial r}\left(v_{0} v_{1}\right)+u_{0} \frac{\partial v_{1}}{\partial x}+u_{1} \frac{\partial v_{0}}{\partial x}\right)+\rho_{1}\left(v_{0} \frac{\partial v_{0}}{\partial r}\right. \\
& \left.\quad+u_{0} \frac{\partial v_{0}}{\partial x}\right)=-\frac{\partial p_{1}}{\partial r}, \\
& \rho_{0}\left(-i \omega w_{1}+v_{0} \frac{\partial w_{1}}{\partial r}+\frac{v_{0} w_{1}}{r}+u_{0} \frac{\partial w_{1}}{\partial x}\right)=-\frac{i m}{r} p_{1}, \\
& \rho_{0}\left(-i \omega T_{1}+v_{0} \frac{\partial T_{1}}{\partial r}+u_{0} \frac{\partial T_{1}}{\partial x}+v_{1} \frac{\partial T_{0}}{\partial r}+u_{1} \frac{\partial T_{0}}{\partial x}\right) \\
& \quad+\rho_{1}\left(v_{0} \frac{\partial T_{0}}{\partial r}+u_{0} \frac{\partial T_{0}}{\partial x}\right)-(\gamma-1)\left(-i \omega p_{1}+u_{0} \frac{\partial p_{1}}{\partial x}+v_{0} \frac{\partial p_{1}}{\partial r}\right. \\
& \left.\quad+u_{1} \frac{\partial p_{0}}{\partial x}+v_{1} \frac{\partial p_{0}}{\partial r}\right)=0 \\
& p_{1} / p_{0}=\rho_{1} / \rho_{0}+T_{1} / T_{0},
\end{aligned}
$$

where $u, v$, and $w$ are the velocities in the axial, radial and azimuthal directions, respectively, and the subscript $m$ has been suppressed.

The boundary conditions are based on the assumption that the duct wall is lined with a point-reacting acoustic material whose specific acoustic admittance $\beta$ may vary along the duct. For no-slip mean flows, a requirement of continuity of the particle displacement gives

$$
v_{1}-R^{\prime} u_{1}=\left(\beta / \rho_{w} c_{w}\right) p_{1}\left(1+R^{\prime 2}\right)^{1 / 2}, \text { at } r=R,
$$

where $R^{\prime}$ is the slope of the wall and the subscript $w$ refers to values at the wall.

An approximate solution to Eqs. (3)-(9) is sought in the form

$$
\begin{aligned}
p_{1} \approx & \sum_{n=1}^{N}\left[A_{n}(x) \psi_{n}^{p}(r, x) \exp \left(i \int k_{n}(x) d x\right)\right. \\
& \left.+\tilde{A}_{n}(x) \tilde{\psi}_{n}^{p}(r, x) \exp \left(i \int \tilde{k}_{n}(x) d x\right)\right],
\end{aligned}
$$

$$
\begin{aligned}
u_{1} \approx & \sum_{n=1}^{N}\left[A_{n}(x) \psi_{n}^{u}(r, x) \exp \left(i \int k_{n}(x) d x\right)\right. \\
& \left.+\tilde{A}_{n}(x) \tilde{\psi}_{n}^{u}(r, x) \exp \left(i \int \tilde{k}_{n}(x) d x\right)\right],
\end{aligned}
$$

with similar expressions for $v_{1}, w_{1}, T_{1}$, and $\rho_{1}$, where the tilde refers to upstream propagation, the $\psi_{n}(r, x)$ are the quasi-parallel mode shapes corresponding to the quasi-parallel propagation coefficients $k_{n}(x)$, and the $A_{n}(x)$ are complex functions whose moduli and arguments represent, in some sense, the amplitudes and the phases of the $(m, n)$ modes. The circumferential mode number $m$ is assumed to be specified and the corresponding subscript on $A, \psi$, and $k$ is not explicitly stated; each variable is expressed as a summation over a finite number of radial modes $n$, with $n=1$ denoting the fundamental radial mode rather than the conventional $n=0$. Since $k_{n}$ is complex, the exponential factor con-, tains an estimate of the attenuation of the $(m, n)$ mode as well as the axial oscillations of the acoustic modes.

Since the $\psi_{n}$ are the quasi-parallel mode shapes, they are the solutions of the following problem:

$$
\begin{aligned}
& -i \hat{\omega} \psi^{p}+i k \rho_{0} \psi^{u}+\frac{i \rho_{0} m}{r} \psi^{w}+\frac{1}{r} \frac{\partial}{\partial r}\left(r \rho_{0} \psi^{\nu}\right)=0, \\
& -i \rho_{0} \hat{\omega} \psi^{u}+\rho_{0} \frac{\partial u_{0}}{\partial r} \psi^{v}+i k \psi^{p}=0, \\
& -i \rho_{0} \hat{\omega} \psi^{v}+\frac{\partial \psi^{p}}{\partial r}=0, \\
& -i \rho_{0} \hat{\omega} \psi^{w}+\frac{i m}{r} \psi^{p}=0, \\
& -i \rho_{0} \hat{\omega} \psi^{T}+\rho_{0} \frac{\partial T_{0}}{\partial r} \psi^{v}+i(\gamma-1) \hat{\omega} \psi^{p}=0, \\
& \psi^{p} / p_{0}=\psi^{p} / \rho_{0}+\psi^{T} / T_{0}, \\
& \psi^{v}-\left(\beta / \rho_{w} c_{w}\right) \psi^{p}=0, \text { at } r=R,
\end{aligned}
$$

where

$$
\hat{\omega}=\omega-k u_{0} \text {. }
$$

Equations (12)-(19) can be combined to yield the wellknown problem for parallel-duct eigenfunctions:

$$
\begin{aligned}
& \frac{\partial^{2} \psi^{p}}{\partial r^{2}}+\left(\frac{1}{r}+\frac{T_{0}^{\prime}}{T_{0}}+\frac{2 k u_{0}^{\prime}}{\hat{\omega}}\right) \frac{\partial \psi^{p}}{\partial r}+\left(\frac{\hat{\omega}^{2}}{T_{0}}-k^{2}-\frac{m^{2}}{r^{2}}\right) \psi^{p}=0, \\
& \frac{\partial \psi^{p}}{\partial r}-i \frac{\omega \beta}{T_{w}^{1 / 2}} \psi^{p}=0, \text { at } r=R .
\end{aligned}
$$

At each axial location, the solution of Eqs. (20) and (21) yields $\psi_{n}^{p}(r ; x)$ and its corresponding propagation coefficient $k_{n}(x)$. The basis functions are normalized so that

$$
\int_{0}^{R} r\left[\psi_{n}^{p}(r ; x)\right]^{2} d r=1 .
$$

Then, Eqs. (12)-(17) are used to express the mode shapes of the other flow variables in terms of $\psi_{n}^{p}$ and $k_{n}$.

Since the transverse dependence in the assumed solution is chosen a priori, it cannot satisfy Eqs. (3)-(9) 
exactly. Thus the assumed solution is constrained by the solvability condition. The deviations from the quasi-parallel solution are required to be orthogonal to every solution of the adjoint-parallel problem. ${ }^{11}$ These constraints yield the following $2 N$ equations for the $A$ 's:

$$
\sum_{n=1}^{2 N} f_{m n} \frac{d A_{n}}{d x}=\sum_{n=1}^{2 N} g_{m n} A_{n},
$$

where $m=1,2,3, \ldots, 2 N$,

$$
\begin{aligned}
f_{m n}= & \left(\int_{0}^{R}\left\{\phi_{1 m}\left(\rho_{0} \psi_{n}^{u}+u_{0} \psi_{n}^{p}\right)+\phi_{2 m}\left(\rho_{0} u_{0} \psi_{n}^{u}+\psi_{n}^{p}\right)+\phi_{3 m}\left(\rho_{0} u_{0} \psi_{n}^{v}\right)+\phi_{4 m} \rho_{0} u_{0} \psi_{n}^{w}+\phi_{5 m}\left[\rho_{0} u_{0} \psi_{n}^{T}-(\gamma-1) u_{0} \psi_{n}^{p}\right]\right\} d r\right) e^{i j k_{n} d x},(24) \\
g_{m n}= & -\left(\int _ { 0 } ^ { R } \left\{\phi_{1 m}\left(\frac{\partial}{\partial x}\left(\rho_{0} \psi_{n}^{u}+u_{0} \psi_{n}^{\rho}\right)\right)-r v_{0} \psi_{n}^{\rho} \frac{\partial}{\partial r}\left(\frac{\phi_{1 m}}{r}\right)+\phi_{2 m}\left[\rho_{0} \frac{\partial}{\partial x}\left(u_{0} \psi_{n}^{u}\right)+\psi_{n}^{\rho}\left(u_{0} \frac{\partial u_{0}}{\partial x}+v_{0} \frac{\partial u_{0}}{\partial r}\right)+\frac{\partial \psi_{n}^{p}}{\partial x}\right]-\psi_{n}^{u} \frac{\partial}{\partial r}\left(\rho_{0} v_{0} \phi_{2 m}\right)\right.\right. \\
& +\phi_{3 m}\left[\rho_{0} u_{0} \frac{\partial \psi_{n}^{v}}{\partial x}+\rho_{0} \psi_{n}^{u} \frac{\partial v_{0}}{\partial x}+\psi_{n}^{\rho}\left(v_{0} \frac{\partial v_{0}}{\partial r}+u_{0} \frac{\partial v_{0}}{\partial x}\right)\right]-v_{0} \psi_{n}^{v} \frac{\partial}{\partial r}\left(\rho_{0} \phi_{3 m}\right)+\phi_{4 m}\left(\frac{\rho_{0} v_{0} \psi_{n}^{w}}{r}+\rho_{0} u_{0} \frac{\partial \psi_{n}^{w}}{\partial x}\right)-\psi_{n}^{w} \frac{\partial}{\partial r}\left(\rho_{0} v_{0} \phi_{4 m}\right) \\
& +\phi_{5 m}\left[\rho_{0} u_{0} \frac{\partial \psi_{n}^{T}}{\partial x}+\rho_{0} \psi_{n}^{u} \frac{\partial T_{0}}{\partial x}+\psi_{n}^{\rho}\left(v_{0} \frac{\partial T_{0}}{\partial r}+u_{0} \frac{\partial T_{0}}{\partial x}\right)-(\gamma-1)\left(u_{0} \frac{\partial \psi_{n}^{p}}{\partial x}+\psi_{n}^{u} \frac{\partial p_{0}}{\partial x}+\psi_{n}^{v} \frac{\partial p_{0}}{\partial r}\right)\right]-\psi_{n}^{T} \frac{\partial}{\partial r}\left(\rho_{0} v_{0} \phi_{5 m}\right) \\
& +(\gamma-1) \psi_{n}^{p} \frac{\partial}{\partial r}\left(v_{0} \phi_{5 m}\right)+i \phi_{1 m}\left(k_{n}-k_{m}\right)\left(\rho_{0} \psi_{n}^{u}+u_{0} \psi_{n}^{p}\right)+i \phi_{2 m}\left(k_{n}-k_{m}\right)\left(\psi_{n}^{p}+\rho_{0} u_{0} \psi_{n}^{u}\right)+i \phi_{3 m}\left(k_{n}-k_{m}\right) \rho_{0} u_{0} \psi_{n}^{v} \\
& \left.\left.+\phi_{4 m} i\left(k_{n}-k_{m}\right) \rho_{0} u_{0} \psi_{n}^{w}+i \phi_{5 m}\left(k_{n}-k_{m}\right) u_{0}\left[\rho_{0} \psi_{n}^{T}-(\gamma-1) \psi_{n}^{P}\right]\right\} d r+\rho_{0} \phi_{1 m}\left(R^{\prime} \psi_{n}^{u}+\frac{\beta}{\rho_{w} c_{w}} \psi_{n}^{p}\left[\left(1+R^{\prime 2}\right)^{1 / 2}-1\right]\right)\right)_{r=R}^{i s k_{n} d x} .
\end{aligned}
$$

For convenience the upstream modes are now denoted by $A_{n}, n=N+1, \ldots, 2 N$, that is

$$
A_{N+n}=\bar{A}_{n}, n=1,2,3, \ldots, N .
$$

Here, the $\phi_{n m}$ are solutions of the adjoint problem; they are related to $\psi_{n}^{p}$ by

$$
\begin{aligned}
& \phi_{1 n}=\frac{r T_{0} \psi_{n}^{p}}{\hat{\omega}}, \quad \phi_{2 n}=\frac{k}{\hat{\omega}} \phi_{1 n}, \quad \phi_{3 n}=\frac{i r T_{0}}{\hat{\omega}^{2}} \frac{\partial}{\partial r}\left(\frac{\omega \phi_{1 n}}{r T_{0}}\right), \\
& \phi_{4 n}=\frac{m \phi_{1 n}}{r \hat{\omega}}, \quad \phi_{5 n}=\frac{\phi_{1 n}}{T_{0}}, \quad \phi_{6 n}=-i \rho_{0} \hat{\omega} \phi_{1 n}
\end{aligned}
$$

\section{RESULTS}

All the results presented here are for the converging circular duct shown in Fig. 1. The duct radius is assumed to vary with axial distance according to

$$
R=1+0.15[-1+\cos (2 \pi x / L)],
$$

where $L$ is the length of the duct. Thus the radius of the duct decreases sinusoidally from 1 in the uniform section to 0.7 at the throat. We will use the sign convention that the mean Mach number is negative or positive if the flow is from right to left (i.e., from the throat to the uniform section) or left to right, respectively.

To evaluate the coefficients $f_{m n}$ and $g_{m n}$ of the set of ordinary differential equations for the mode amplitudes $A_{n}$, one must specify all mean-flow variables $u_{0}, \rho_{0}$, $T_{0}, p_{0}, v_{0}$, and their first partial derivatives with respect to both $x$ and $r$. The form of $g_{m n}$ as given in Eq. (25) would also require some second derivatives. How ever, those terms involving second derivatives have been integrated by parts and the resulting expression for $g_{m n}$, which requires only first derivatives of the mean flow, has been used in the numerical development.

Although the program is general and can accept any mean flow, a simplified model of the mean flow has been employed in the present study because the effort involved in calculating the actual mean profiles is substantial. This model uses one-dimensional gas-dynamics theory to describe the mean-flow variables in the inviscid core; thus $u_{0}, p_{0}, T_{0}$, and $\rho_{0}$ are constant across the duct cross section except in the region of the wall boundary layer. The radial velocity $v_{0}$ is calculated to be a linear function of $r$ consistent with the mean-continuity equation and the flow tangency condition at the wall. The wall is assumed to be adiabatic and the temperature profile is assumed to be related to the velocity profile by

$$
T_{0} / T_{c}=1+\frac{1}{2} r_{1}(\gamma-1) M_{c}^{2}\left[1-\left(u_{0} / u_{e}\right)^{2}\right],
$$

where the subscript $c$ refers to values in the inviscid core, $r_{1}=0.8$ is the recovery factor, and $\gamma=1.4$ is the ratio of the gas specific heats. Specification of the mean Mach number and displacement thickness at $x=.0$ permits their subsequent axial variations to be calculated from mass-flow considerations. The axial variations of $T_{c}, \rho_{c}, u_{c}$, etc. are computed from one-dimensional gas-dynamics theory and then the boundary-layer profiles are computed. In this study a quadratic boundary-layer profile is used. For a lined duct, the liner is taken to be uniform and point reacting so that it can be represented by a nondimensional admittance $\beta$ that is independent of $x$ in the form

$$
\beta=\left\{R_{e}\left(1-i \omega / \omega_{0}\right)+i \cot \left[\omega d /\left(T_{i}\right)^{1 / 2}\right]\right\}^{-1},
$$

where $R_{e}$ is the resistance of the facing sheet, $\omega_{0}$ is the characteristic frequency of the facing sheet, and $d$ is the depth of the backing cavity of the liner. Here,

$$
T_{w}=T_{a d}=T_{c}\left[1+\frac{1}{2}(\gamma-1) r_{1} M_{c}^{2}\right] .
$$

In the inviscid core Eq. (20) reduces to Bessel's equation

$$
\frac{\partial^{2} \psi^{p}}{\partial r^{2}}+\frac{1}{r} \frac{\partial \psi^{p}}{\partial r}+\left(\frac{\hat{\omega}^{2}}{T_{0}}-k^{2}-\frac{m^{2}}{r^{2}}\right) \psi^{p}=0,
$$


so that $\psi^{p}$ can be expressed as

$$
\psi_{n}^{p}(r, x)=A_{n}(x) J_{m}\left(\kappa_{m, n} \gamma\right)+B_{n}(x) Y_{m}\left(\kappa_{m, n} \gamma\right)
$$

at each axial station where $J_{m}$ and $Y_{m}$ are Bessel functions of order $m$ of the first and second kinds, respectively, and $\kappa_{m, n}$ is the quasi-parallel eigenvalue and is related to the wavenumber by the following equation:

$$
\kappa_{m, n}^{2}=\hat{\omega}^{2} / T_{0}-k_{m, n}^{2} .
$$

For $\psi_{n}^{p}$ to be bounded at $r=0, B(x)$ must be zero since $Y_{m}(0)$ is unbounded. Using the above facts we implemented the following scheme to determine the quasiparallel wavenumbers and eigenfunctions. First an initial guess is made for the wavenumber so that in the inviscid core

$$
\psi_{n}^{p}=J_{m}\left(\kappa_{m, n} r\right), \quad 0 \leqslant r<R-\delta,
$$

where $\delta$ is the boundary-layer thickness. Now the values of $\psi_{n}^{p}$ and $\partial \psi_{n}^{p} / \partial r$, evaluated at $r=R-\delta$, are used as initial conditions to integrate Eq. (20) to the duct wall. Once at $r=R$ an iteration procedure is begun on Eq. (21) to determine the correct wavenumber.

The specific numerical methods used are a fourthorder Runge-Kutta integration routine and a modification of the Levenberg-Marquardt algorithm. ${ }^{12,13}$ The Levenberg-Marquardt algorithm minimizes the sum of the squares of $M$ functions in $N$ variables. With the initial wavenumber, the integration is implemented on Eq. (20) with the initial conditions

$$
\begin{aligned}
& \psi_{n}^{p}=J_{m}\left(\kappa_{m, n} \gamma\right), \quad r=R-\delta, \\
& \frac{\partial \psi_{n}^{p}}{\partial r}=\kappa_{m, n} J_{m}^{\prime}\left(\kappa_{m, n} r\right), \quad r=R-\delta,
\end{aligned}
$$

where $\kappa_{m, n}$ is a function of the wavenumber $k_{m, n}$. Now at the duct wall the boundary condition is evaluated, and here a complex function $F\left(k_{m, n}\right)$ is defined as

$$
F\left(k_{m, n}\right)=\frac{\partial \psi_{n}^{p}}{\partial r}-i \frac{\omega \beta}{T_{w}^{1 / 2}} \psi_{n}^{p} .
$$

Since $F$ and $k_{m, n}$ are complex, Eq. (38) can be separated into two real equations in two real unknowns. The function $\left|F\left(k_{m, n}\right)\right|^{2}$ is minimized using the Levenberg-Marquardt algorithm. This procedure is done iteratively until $\left|F\left(k_{m, n}\right)\right| \approx 0$. A subroutine that is able to evaluate Bessel functions of a complex argument is supplied to the model. Therefore the need to integrate Eq. (20) across the inviscid core is eliminated.

This method also provides consistent initial conditions, Eqs. (36) and (37), for the integration of Eq. (20). If $m=0$ the flow is axisymmetric (no $\theta$ dependence) and if $m \neq 0$, then a particular spinning mode is analyzed. A note of caution is emphasized here concerning the wavenumbers. They should be examined throughout the duct to eliminate the possibility of the development of any exponentially amplifying modes. This would violate the basic idea that the quasi-parallel wavenumbers, $k_{m, n}$, are the wavenumbers that would exist in an infinite parallel duct, since they would become unbounded. If this does occur then that particular mode should be dropped in Eqs. (10), (11), etc.

Figures 2 and 3 compare the acoustic pressure pro-
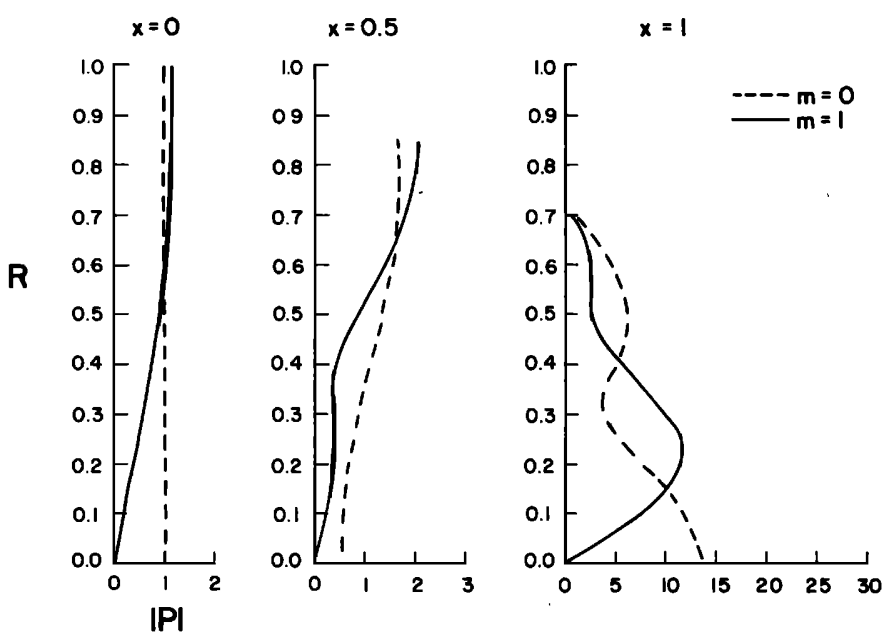

FIG. 2. Comparison of the acoustic pressure profiles at $x$ $=0,0.5$, and 1 in a hard-walled duct for $m=0$ and 1 when the first upstream mode is incident at $x=0, \omega=9, M_{t}=-0.883$, $\delta_{0}^{*}=0.001$, and $N=4$.

files for an axisymmetric mode and three spinning modes $(m=1,2,3)$ in a hard-wall duct. The input for these cases is the same except for the circumferential (spinning) mode number. The profiles for three axial stations are presented $(x=0,0.5,1)$. In all the results presented the throat of the converging duct is at $x=1$. The throat mean Mach number is -0.883 , the inlet displacement thickness is 0.001 , the frequency is 9 , and four modes are considered. At $x=0$, the lowest right-running mode is incident and it has the same value in all cases so that the acoustic signal propagates upstream. As is expected the maximum pressure amplitude in the axisymmetric case occurs at the centerline of the throat. With increasing spinning mode number one sees that the maximum amplitude decreases and shifts toward the duct wall at the throat. This indicates that whereas the refractive effect of the axial gradients is still present the focusing and intensification is weakened by the asymmetries.

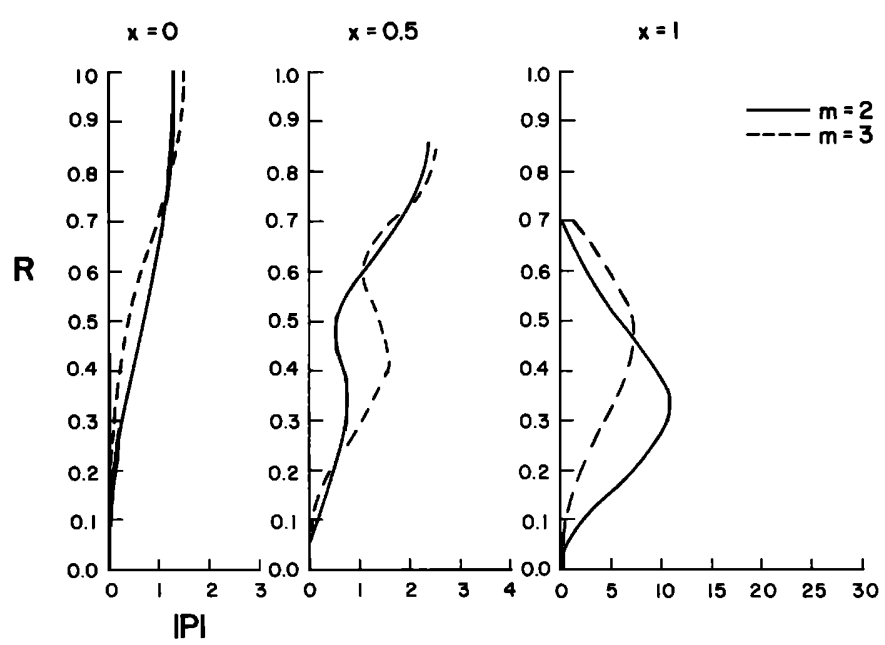

FIG. 3. Comparison of the acoustic pressure profiles at 0 , 0.5 , and 1 in a hard-walled duct for $m=2$ and 3 where the first upstream mode is incident at $x=0, \omega=9, M_{t}=-0.883, \delta_{0}^{*}$ $=0.001$, and $N=4$. 

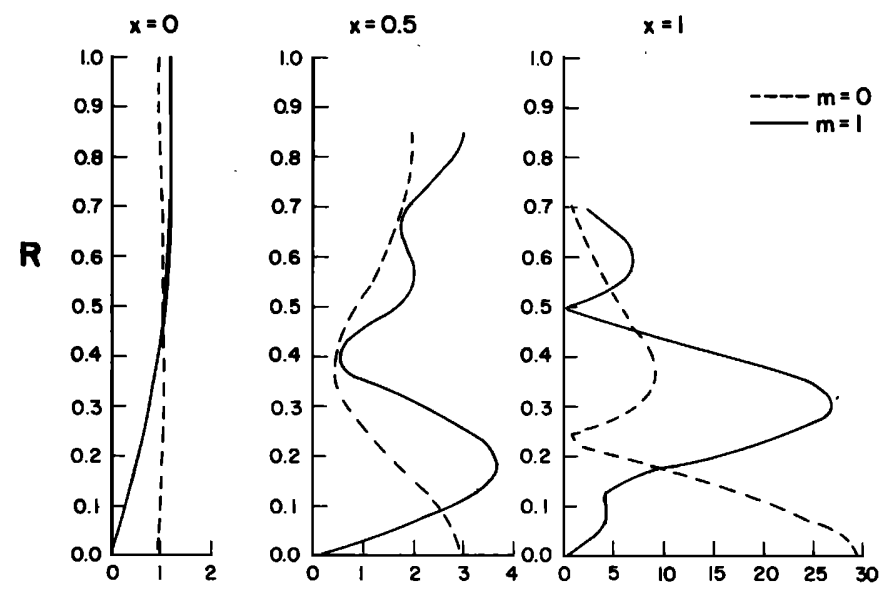

IPI

FIG. 4. Comparison of the acoustic pressure profiles at $x$ $=0,0.5$, and 1 in a hard-walled duct for $m=0$ and 1 where the first upstream mode is incident at $x=0, \omega=9, M_{t}=-0.883$, $\delta_{0}^{*}=0.001$, and $N=7$.

Figures 4 and 5 depict the same cases except for the fact that the number of modes has been increased to seven. The decrease in amplitude and its shift toward the wall at the throat are still present. But there is a noticeable difference between the pressure profiles for the cases of $m=0$ and $m=1$ as compared to the cases using four modes. For $m=2$ and $m=3$ the profiles are little changed by considering seven modes. These differences can be explained by examining the quasi-parallel wavenumbers. The first four wavenumbers in each case are cut on throughout the duct, but the next three can contain modes that are initially cut off. In the axisymmetric case only one cutoff mode is added, for $m=1$ two are added and for both $m=2$ and $m=3$ all three modes are cut off. Also as the spinning mode number increases the attenuation factors increase. Therefore, for the cases of $m=2$ and $m=3$, these modes are greatly attenuated as compared to $m=0$ and $m=1$. It can also be seen that for each case the inclusion of seven modes results in a greater peak pressure at the throat.
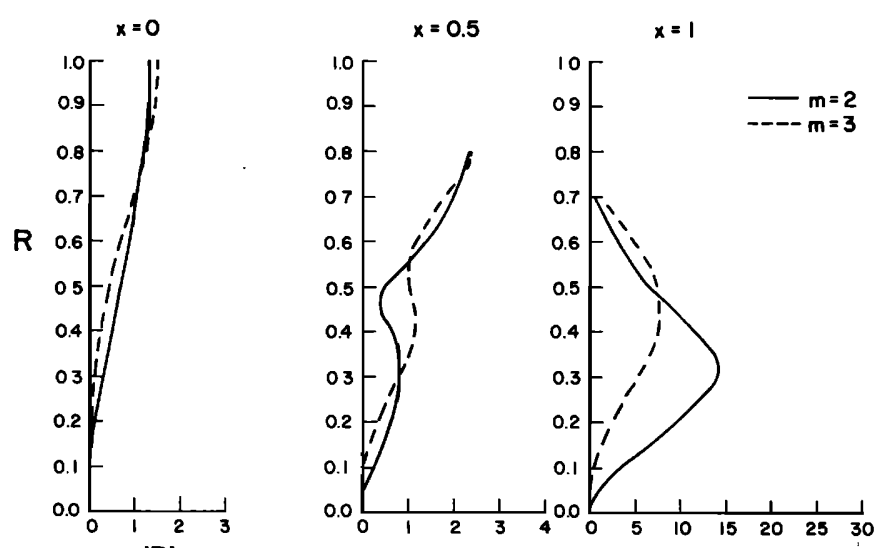

FIG. 5. Comparison of the acoustic pressure profiles at $x$ $=0,0.5$, and 1 in a hard-walled duct for $m=2$ and 3 when the first upstream mode is incident at $x=0, \omega=9, M_{t}=-0.883, \delta_{0}^{*}$ $=0.001$, and $N=7$.

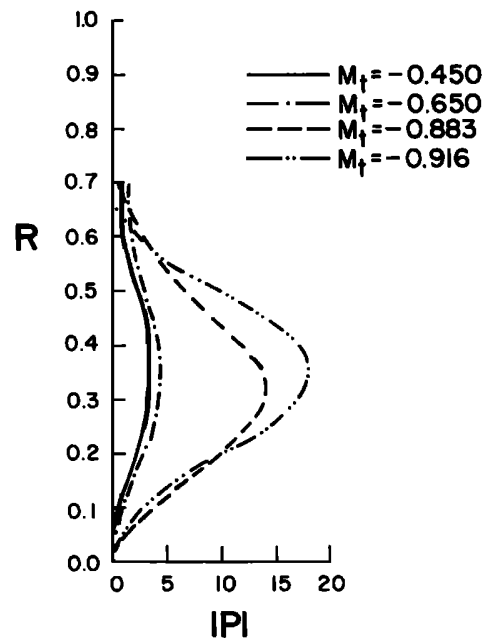

FIG. 6. Effect of mean Mach number on the acoustic pressure profile at the throat of a hard-walled duct for $m=2$ when the first upstream mode is incident at $x=0, \omega=9, \delta_{0}^{*}=0.001$, and $N=7$.

Figures 6 and 7 show the effect of the mean Mach number at the throat. The input parameters are the same as those used to construct the previous figures except for the Mach number variations. Figure 6 depicts the case for $m=2$ and Fig. 7 for $m=3$. Seven modes were included in each of these cases. They show the increase in the acoustic pressure amplitude as the throat Mach number increases toward unity. For partially choked flows one should be aware of the limitations in using a linear model. As the mean Mach number increases the wavenumbers of the right-running (upstream) modes become very large. Consider the case for uniform flow where the wavenumber is given by

$$
k=\left\{-\omega M_{c} \mp\left[\omega^{2}-\left(1-M_{c}^{2}\right) \kappa^{2}\right]^{1 / 2}\right\} /\left(1-M_{e}^{2}\right) .
$$

Here $M_{c}$ is the Mach number and $\kappa$ is the eigenvalue. As $M_{c} \rightarrow 1$, one of the values of $k$ approaches infinity,

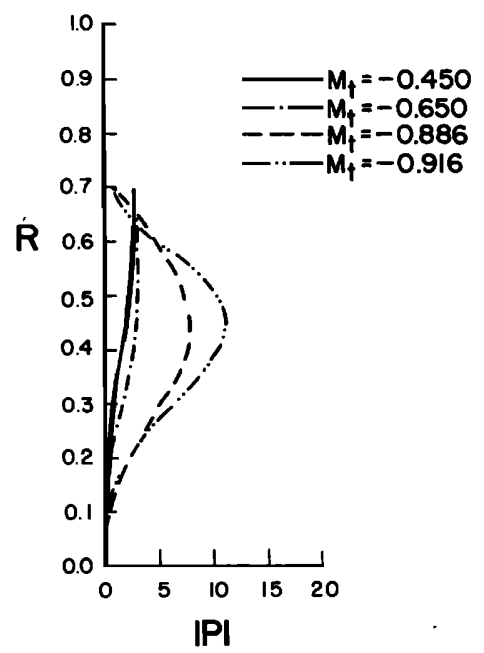

FIG. 7. Effect of mean Mach number on the acoustic pressure profile at the throat of a hard-walled duct for $m=3$ when the first upstream mode is incident at $x=0, \omega=9, \delta_{0}^{*}=0.001$, and $N=7$. 


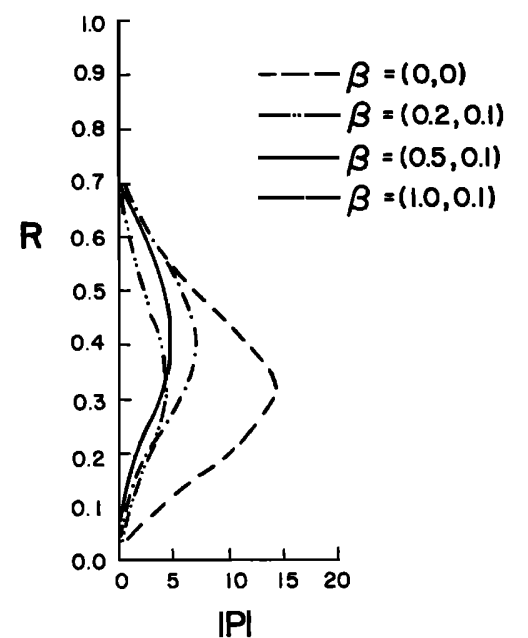

FIG. 8. Effect of admittance on the acoustic pressure profile at the throat of a lined duct for $m=2$ when the first upstream mode is incident at $x=0, M_{t}=-0.883, \omega=9, \delta_{0}^{*}=0.001$, and $N=7$.

whereas the other remains bounded. This unusual behavior of the solution is due to linearization of the acoustic equations, as demonstrated numerically by Eisenberg and $\mathrm{KaO}^{14}$ and Hersh and $\mathrm{Liu}^{15}$ and analytically by Myers and Callegari ${ }^{16}$ for the one-dimensional case. Thus the inclusion of the nonlinear terms becomes necessary at high Mach numbers. ${ }^{17-19}$

The effect of the linear admittance, $\beta$, is demonstrated in Figs. 8 and 9 for $m=2$ and $m=3$. The acoustic pressure profiles at the throat are shown for four values of the admittance for each spinning mode. The throat Mach number is -0.883 , the inlet displacement thickness is 0.001 , the frequency is 9 , and seven modes are considered. The real part of the admittance is varied and the lowest right-running mode is input at $x=0$ so that upstream propagation takes place. As is shown there is a significant reduction in the amplitude of the acoustic signal if the duct is lined, especially

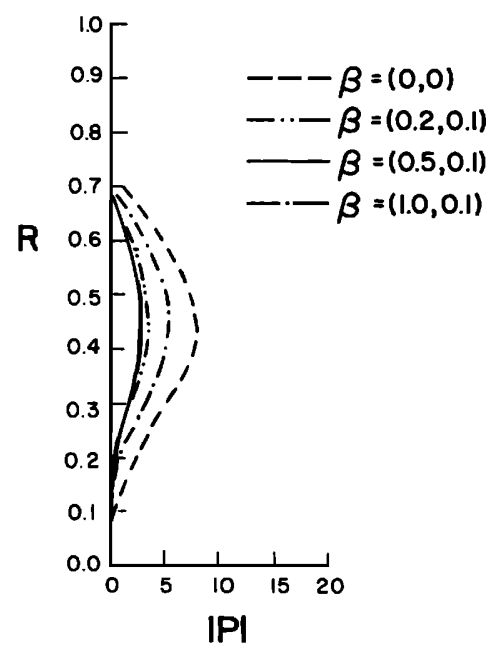

FIG. 9. Effect of admittance on the acoustic pressure profile at the throat of a lined duct for $m=3$ where the first upstream mode is incident at $x=0, M_{t}=-0.883, \omega=9, \delta_{0}^{*}=0.001$, and $N=7$.

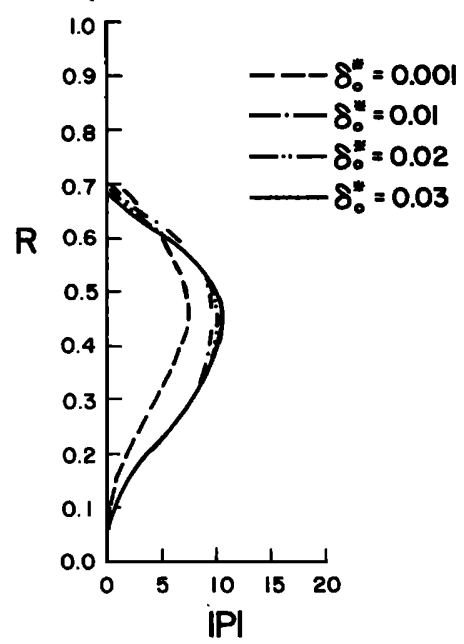

FIG. 10. Effect of boundary-layer thickness on the acoustic pressure profile at the throat of a hard-walled duct for $m=3$ where the first upstream mode is incident at $x=0, M_{t}=-0.883$, $\omega=9$, and $N=4$.

for $m=2$. No definite trend in the amplitude reduction as a function of the real part of the liner admittance is apparent in contrast to the axisymmetric case. ${ }^{3}$

The effect of transverse velocity (shear layers) and temperature gradients of the mean flow on the propagation and attenuation of sound waves in hard-walled as well as lined rectangular, circular, and annular ducts have been investigated in several studies. ${ }^{6}$ In general, the shear layers refract the axisymmetric modes toward the wall for downstream propagation and away from the wall for upstream propagation. The degree of refraction tends to increase with increasing frequency and increasing boundary-layer thickness. Cooling the wall of a duct tends to refract the sound toward the wall for both upstream and downstream propagation, whereas heating the wall tends to refract the sound away from the wall.

Figure 10 shows the effect of boundary-layer thickness for $m=3$ in a hard-wall duct. The acoustic pressure profiles presented were calculated at the throat and the relevant physical parameters are mean throat Mach number $=-0.883$, frequency $=9$, and four modes.

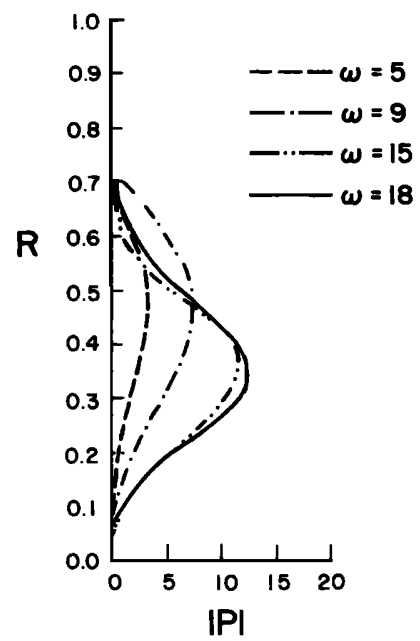

FIG. 11. Effect of the acoustic frequency on the acoustic pressure profile at the throat of a hard-walled duct for $m=3$ where the first upstream mode is incident at $x=0, M_{t}=-0.883$, $\delta_{0}^{*}=0.001$, and $N=4$. 

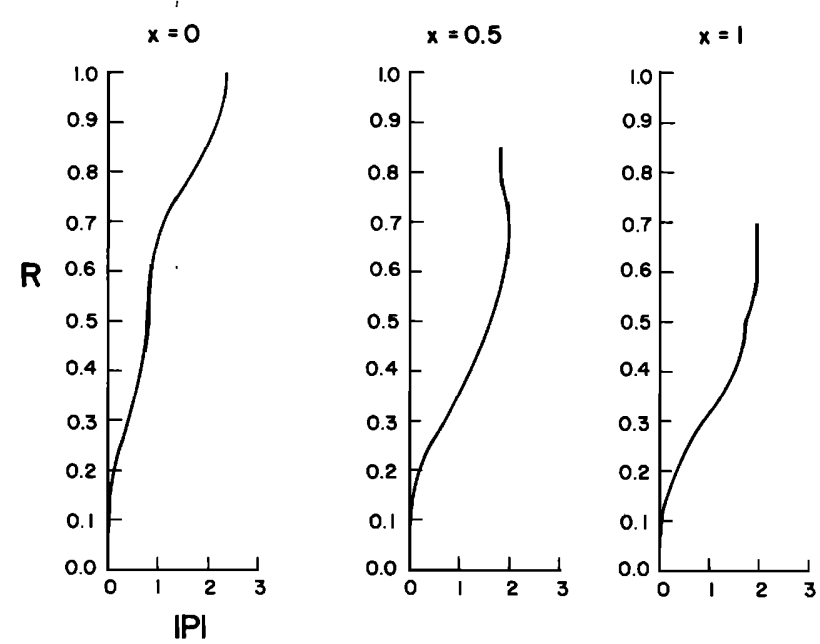

FIG. 12. Acoustic pressure profiles at $x=0,0.5$, and 1 in a hard-walled duct for $m=3$ when the first downstream mode is incident at $x=1, M_{\mathrm{t}}=-0.883, \delta_{0}^{*}=0.001$, and $N=7$.

The lowest right-running mode is incident at $x=0$ so that the acoustic signal propagates upstream. As the inlet displacement thickness increases the peak acoustic pressure amplitude increases and the wall value decreases. Therefore it appears that the boundarylayer thickness has a strong refractive effect for spinning modes as well as axisymmetric modes. But as noted before the signal is not focused along the duct centerline.

The dependence on the frequency of the acoustic pressure is shown in Fig. 11. The input values for this case are the same as the previous case except that the inlet displacement thickness is set at 0.001 and the frequency is varied. Again the plots are constructed at the throat and four modes are included. The lowest right-running mode is incident at $x=0$ which implies that upstream propagation takes place. Increasing the frequency results in an increase in the amplitude of the maximum acoustic pressure.

Figure 12 represents a case where the acoustic signal propagates downstream in a hard-wall duct. The lowest left-running mode is incident at $x=1$ and seven modes are considered. The mean throat Mach number is $-0.883, m=3$, the frequency is 9 , and the inlet displacement thickness is 0.001 . Acoustic pressure profiles are shown at the three axial stations $x=0,0.5$, and 1. Note that there is only a slight increase in the maximum acoustic pressure as the signal propagates downstream. It also appears that at $x=0$ the signal has undergone significant refraction toward the wall where the maximum amplitude occurs in spite of the rather small boundary-layer thickness. Since there is no appreciable growth in the acoustic pressure here resulting in the solution becoming unstable one suspects that the li- near theory is applicable to cases of waves propagating with the flow even if the mean Mach number is high.

\section{ACKNOWLEDGMENT}

This work was supported by the NASA-Langley Research Center under Grant No. NSG-1573.

${ }^{1}$ F. B. Greatrex, "By-pass engine noise," Trans. SAE 69, 312-324 (1961).

${ }^{2}$ J. A. Sobel and A. D. Welliver, "Sonic block silencing for axial and screw-type compressors," Noise Control 7, 9-11 (1961).

${ }^{3}$ A. H. Nayfeh, "Intensification and refraction of acoustic signals in partially-choked converging ducts," J. Sound Vib. $73,519-531$ (1980).

${ }^{4}$ A. H. Nayfeh, J. E. Kaiser, and B. S. Shaker, "A waveenvelope analysis of sound propagation in nonuniform circular ducts with compressible mean flows," NASA Contractor Rep. 3109 (1979).

${ }^{5}$ A. H. Nayfeh, B. S. Shaker, and J. E. Kaiser, "Transmission of sound through nonuniform circular ducts with compressible mean flows," AIAA J. 18, 515-525 (1980).

${ }^{6}$ A. H. Nayfeh, J. E. Kaiser, and D. P. Telionis, "Acoustics of aircraft engine-duct systems," AIAA J. 13, 130-153 (1975).

${ }^{7}$ A. H. Nayfeh, "Sound propagation through nonuniform ducts," NASA CP-2001 3, 821-833 (1976).

${ }^{8}$ R. K. Sigman, R. K. Majjigi, and B. T. Zinn, "Determination of turbofan inlet acoustics using finite elements," AIAA J. 16, 1139-1145 (1978).

${ }^{9}$ W. Eversman, R. J. Astley, and V. P. Thanh, "Transmission in nonuniform ducts-a comparative evaluation of finite element and weighted residuals computation schemes," AIAA Paper 77-1299 (1977).

${ }^{10} \mathrm{~A}$. L. Abrahamson, "A finite element model of sound propagation in a nonuniform circular duct containing compressible flow," AIAA Paper 77-1301 (1977).

${ }^{11} \mathrm{~A}$. H. Nayfeh, Introduction to Perturbation Techniques (Wiley-Interscience, New York, 1981), Chap. 15.

${ }^{12}$ K. M. Brown and J. E. Dennis, "Derivative free analogues of the Levenberg-Marquardt and Gauss algorithms for nonlinear least squares approximations," Numer. Math. 18, 289-297 (1972)

${ }^{13}$ B. S. Garbow, K. E. Hillstrom, and J. J. More, MINPACK documentation, Argonne National Lab., Argonne, IL (1979).

${ }^{14}$ N. A. Eisenberg and T. W. Kao, "Propagation of sound through a variable-area duct with a steady compressible flow," NASA CR-135123 (1976).

${ }^{15}$ A. S. Hersh and C. Y. Liu, "Sound propagation of sound through a variable-area duct with a steady compressible flow," NASA CR-135123 (1976).

${ }^{16}$ M. K. Myers and A. J. Callegari, "On the singular behavior of linear acoustic theory in near-sonic duct flows," J. Sound Vib. 51, 517-531 (1977).

${ }^{17}$ A. H. Nayfeh, J. J. Kelly, and L. T. Watson, "Nonlinear propagation in near sonic flows," AIAA Paper 80-0096 (1980).

${ }^{18}$ A. H. Nayfeh, B. S. Shaker, and J. E. Kaiser, "Computation of nonlinear one-dimensional waves in near-sonic flows," AIAA J. 16, 1154-1159 (1978).

${ }^{19}$ A. J. Callegari and M. K. Myers, "Sound transmission in ducts containing nearly choked flows," AIAA Paper 79-0623 (1979). 